\title{
SPARSE RADIOGRAPHIC TOMOGRAPHY AND SYSTEM IDENTIFICATION IMAGING FROM SINGLE VIEW, MULTIPLE TIME SAMPLE DENSITY PLOTS
}

\author{
T. J. ASAKI ${ }^{1}$, E. M. BOLLT ${ }^{2}$, AND K. R. VIXIE ${ }^{3}$
}

\begin{abstract}
Tomography is a classic inverse problem in which multiple density projections of an object are processed to infer some approximation of the original. We consider the highly sparse inverse problem of single angle projection, but seek to reduce the ambiguity through multiple time observations in a dynamic system of known or partially known dynamics. In this work we solve the planar problem by optimization techniques based on a gradient-free multi-directional search algorithm to minimize our nonlinear functional. We demonstrate convincingly successful numerical examples to support our relatively simple technique.
\end{abstract}

2000 Mathematics Subject Classification: 65R32, 93B10, 93B30.

Keywords: tomography, dynamics, sparse data, system identification.

\section{Introduction}

We will discuss the following two sparse-data inverse problems.

1. Radiographic tomography. We wish to infer the shape of some unknown simple object which evolves under a known model. We know a general model form of the flow in the plane of initial conditions, which evolves simple regions. We observe data only through single angle projections, but at multiple time shots. We design a functional which rewards close match between a predicted object form whose evolution projects are close to the given data. We minimize the function over a class of test objects using a gradient free scheme.

2. System identification problem. We wish to infer parameters (the physics) of a system by observation of data from some unknown simple object. A general form of the model is assumed, but specific parameters such as values of masses, friction, viscosity, etc., are left as unknown parameters which we must estimate. Thus we have the radiographic tomography problem above together with estimation of parameters.

Previous work on this problem is found mostly in the area of observability and state space reconstruction though a small number of works can be found which mention the dynamic tomography problem $([1-3,23,24])$.

While the problem of tomography was solved by the introduction of the Radon transform and its inverse over 100 years ago, it was not practically solved until the work of Hounsfield

\footnotetext{
${ }^{1}$ Continuum Dynamics (CCS-2), Los Alamos National Laboratory, Los Alamos, New Mexico 87544. Email: asaki@lanl.gov

2 Mathematics Department, Clarkson University, Potsdam NY 13699-5815. E-mail: bolltem@clarkson.edu

${ }^{3}$ Mathematical Modeling and Analysis (T-7), Los Alamos National Laboratory, Los Alamos, New Mexico 87544. E-mail: vixie@lanl.gov
} 
and Cormack (Hounsfield and Cormack shared the 1979 Nobel Prize in Medicine for the invention). Typical tomographic inversions require several hundred angles for the accuracy demanded in medical and manufacturing imaging. However, the tomographic measurement of rapidlyevolving dense objects is characterized by a small number of measurements (a short temporal sequence of a few (1-5) angular projections). For this highly sparse data various simplifying assumptions are usually used. For example, if the objects being observed are cylindrically symmetric, the Abel inversion can be used (see [4] and [5]). While the assumption of cylindrical symmetry can work quite well, there are certainly important cases in which the object is not cylindrically symmetric, and in these cases it makes sense to use all the data over time for the inversion.

Our approach is to formulate the inverse problem variationally using the entire time sequence of data and solve the resulting nonlinear optimization problem by a direct search method. We do this both for the case where the dynamical equations are completely known as well as the case where important parameters which determine the dynamics are unknown and need to be estimated from the data along with the objects being reconstructed.

While there is not much previous work on this problem in a tomographic context, it is very close to the questions of observability and state-space reconstruction $[15,16,18,20,21]$. These papers prove theorems which are quite similar to the theorems in section 3 (and equivalent in the case of the Minimal Reduction Theorem which can be found in [17].) In section 3 we follow [2] which both directly addresses the questions we are concerned with and explains geometrically the key ideas.

\section{Statement of radiographic tomography problem}

We wish to infer the action of a planar flow on a simple region $R$ allowing only projected observations at multiple times as data. First we define these terms.

A planar flow is defined as follows. We assume a model in the form of a continuous dynamical system

$$
\dot{\mathbf{z}}=\mathbf{f}_{\mathbf{p}}(\mathbf{z}), \quad \mathbf{z}(t) \in \mathbb{R}^{2},
$$

where $\mathbf{p}$ represents adjustable parameters (such as mass, coefficients of friction or viscosity, etc.) in the family of models. At first we will think of $\mathbf{p}$ as constant but later which we relax this assumption when we consider the system identification problem in Sec. 6. Assuming an f which gives global existence and uniqueness implies a continuous group of solutions,

$$
\mathbf{z}(t)=\Phi_{t}\left(t ; \mathbf{z}_{0}\right),
$$

which is also called a continuous dynamical system or flow. That is, $\Phi_{t}: \mathbb{R}^{2} \rightarrow \mathbb{R}^{2}$ is a continuously parameterized family of functions.

We define a simple region $R \subset \mathbb{R}^{2}$ to be a finite union of open sets. Therefore, we consider the action of the flow on the entire region

$$
R(t)=\cup_{\mathbf{z} \in R(0)} \Phi_{t}(t ; \mathbf{z}),
$$

where $R(0) \equiv R$ denotes the initial simple region deforming in time under the action of the flow.

We define the term "projection", as a single angle density projection operator,

$$
P: L^{1}\left(\mathbb{R}^{2}\right) \rightarrow L^{1}(\mathbb{R}), \quad \nu(x)=P[\rho(x, y)]=\int_{\mathbb{R}} \rho(x, y) d y,
$$


For simplicity, we will assume that the density is zero outside our regions of interest and uniformly one inside the regions. In more generality, movement, of general density profiles which evolve continuously with the flow $\Phi_{t}: \mathbb{R}^{2} \rightarrow \mathbb{R}^{2}$, is defined through an adjoined dynamical system, $F P_{t}: L^{1}\left(\mathbb{R}^{2}\right) \rightarrow L^{1}\left(\mathbb{R}^{2}\right)$, through the Frobenius — Perron operator $[8,9], \rho(\mathbf{z}, t)=F P_{t}[\rho(\mathbf{z}, 0)]=\int_{\mathbb{R}^{2}} \delta\left(\mathbf{z}-\Phi_{t}(\mathbf{w})\right) \rho(\mathbf{w}, 0) d \mathbf{w}$. We find our simplified specific notation more direct and sufficient for uniform distributions supported on the simple regions. Therefore, we will be loose with our notation and also write

$$
R(t) \in L^{1}\left(\mathbb{R}^{2}\right), \text { denotes, } \mathbf{1}_{R(t)}
$$

by using the characteristic function

$$
\mathbf{1}_{A}(x, y)= \begin{cases}1, & \text { if }(x, y) \subset A \\ 0, & \text { else }\end{cases}
$$

Without loss of generality, we have assumed that the projection is parallel to the $y$-axis, since a change of variables can so rotate any other projection, and such a rotation is a conjugacy of the dynamical system.

The measured data is assumed to be in the form of observed projections. That is, we have several density profiles measured at discrete times, $\left\{D\left(t_{i}\right)\right\}_{i=0}^{n}$, where each $D(t)$ is a density function,

$$
D(t) \in L^{1}(\mathbb{R}) .
$$

We assume that we do not know the shape in question. Our job is to guess the "right" initial test region $R(0)$, so that its evolution is such that its projections best match the measured data at each time. We grade our success compared to the data by the cost functional

$$
J[R(0)]=\sum_{i=0}^{n}\left\|\tilde{D}\left(t_{i}\right)-D\left(t_{i}\right)\right\|_{L^{1}(\mathbb{R})},
$$

where

$$
\tilde{D}\left(t_{i}\right) \equiv P\left[R\left(t_{i}\right)\right]
$$

defines the test data $\tilde{D}\left(t_{j}\right)$ of test region $R\left(t_{j}\right)$ projected at time $t_{j}$ to compare to the measured data $D\left(t_{j}\right)$ at time $t_{j}$. Here, $R(t)$ is the flowing density profile of the region defined through Eqs. (2.3) and (2.5). Assuming the absence of noise and a perfect model, this functional returns zero when the initial shape is found which generates the data. Since neither of these assumptions are physically realizable, we will be happy with a small residual. That is, we wish to minimize $J$ within the class of uniform densities over simple regions. The issue of when there is a nontrivial null space of this functional is discussed in the following section.

\section{Theoretical considerations of observability: how many density profiles are necessary?}

The question of what number of observations are required to reconstruct an object from a time sequence of single-angle measurements is answered by the following theorem valid for nonstationary linear dynamics. 
The context for the theorem is a system $\left\{\left\{O_{i}, m_{i}\right\}_{1}^{k},\left\{\phi_{i}\right\}_{1}^{k-1}, L\right\}$ where the $O_{i} \in R^{n}$ and the $m_{i} \in R^{m}$ are the evolving states (i.e., the evolving 2-dimensional object representation) and the sequence of measurements. We assume that the linear measurement operator $L$ : $R^{n} \rightarrow R^{m}$ is full rank, each $\phi_{i}: R^{n} \rightarrow R^{n}$, that

$$
O_{i+1}=\phi_{i}\left(O_{i}\right), \quad i=1, \ldots, k-1,
$$

and

$$
m_{i}=L\left(O_{i}\right), \quad i=1, \ldots, k .
$$

For any particular dynamical sequence $\left(\left\{\phi_{i}\right\}_{1}^{k-1}\right)$ we will call $\left\{O_{i}\right\}_{1}^{k}$ the inverse of $\left\{m_{i}\right\}_{1}^{k}$ if these two satisfy Eqs. 3.1 and 3.2. We note that, for our case, it is the uniqueness of an inverse which is in question (there are typically many inverses of any realized data sequence).

Theorem 3.1 (Optimal reconstruction theorem). If $k \geqslant\lceil m / n\rceil$, then the set of dynamical sequences $\left\{\phi_{i}\right\}_{1}^{k}$ for which there is a unique inverse is dense in $R^{(k-1) n^{2}}$.

That is equivalent to theorem 6.2 in [2].

In fact, we can always get a unique inverse if we have enough measurements (enough is always less than $n$ ) provided that the $\phi_{i}$ do not map $N_{L}$, the null-space of $L$, or any of its subspaces back into themselves. If there are preserved subspaces, then there are nontrivial null sequences, $\left\{n_{i}\right\}_{1}^{k} \in N_{L}$ such that

$$
n_{i+1}=\phi_{i}\left(n_{i}\right), \quad i=1, \ldots, k-1,
$$

and, of course,

$$
0=L\left(n_{i}\right), \quad i=1, \ldots, k .
$$

These null sequences can be added to any inverse of a particular data sequence to get another, distinct inverse. The Minimal Reduction Theorem of [2] states that if there is no special relationship between the dynamics (the $\phi$ ), and the null space of $L, N_{L}$, then we will, in at most $n$ measurements, gain uniqueness of inverses.

The essential intuition is that since submanifolds of a state space typically, or generically, intersect transversely, we expect that the number of observations that are needed to recover the state is simply the number of degrees of freedom in the state space divided by the number of degrees of freedom in the measurement. In the stochastic case there are other factors which are related to the strengths of the instabilities in the dynamics which govern how quickly the uncertainty diffuses.

The theory is closely related to observability results in control theory and phase space reconstructions (delay coordinate embeddings) in dynamical systems. Our results are different in that we consider variations of the dynamics with the observation function kept fixed, whereas other results either assume that the dynamics are fixed and the observation function changes or that both the dynamics and the observation function are variable, (see $[15,16,18,20,21])$. While Aeyels [15] does consider the case where the observation function is fixed and the dynamics are variable he does so for vector fields (not maps). He is also looking at the case where he wants all initial points to be recoverable from the sequence of measurements and this requires $2 n+1$ 1-dimensional measurements to recover the $n$-dimensional initial points. Similar comments apply to the comparison to Stark's more recent paper [20]. Our minimal reduction theorem is a more precise version of the well-known theorem in control theory that states that if the observability matrix is not full rank, then no number of measurements can give you full information on the state of the system and 
if it is full rank, then you need at most $n$ measurements (of any dimension) of a system that has an $n$-dimensional state space. In the preparation of this paper we became aware of theorem 5.3.13 in [17] which, together with the usual observability theorem, is equivalent to our minimal reduction theorem.

\section{Numerical techniques for radiographic tomography problem}

4.1. Multidirectional search algorithm (MDSA) for unconstrained optimization. As formulated, our problem reduces to a high-dimensional unconstrained optimization problem for which we do not have explicit derivatives. We have enlisted the "Multidirectional search algorithm" (MDSA) [10,11] which we discuss here briefly. MDSA is a highly robust general purpose optimization algorithm which is especially powerful for strictly convex cost functions, $f: \mathbb{R}^{n} \rightarrow \mathbb{R}$ with global minimum $x^{*}$. The goal of any unconstrained optimization problem is to minimize $f(x), x \in \mathbb{R}^{n}$. MDSA is a gradient-free method which is proven in [10] to converge to at least a local minimum if $f \in C^{1}$ and to a global minimum if the cost function is also strictly convex. Popular competitor algorithms, such as the Nedler-Mead simplex method [13] which also does not require explicit gradients of $f$, cannot guarantee global minimization for strict convexity.

MDSA builds $n$-simplices, on whose vertices, the cost function $f$ is evaluated, followed by decisions as to which vertices are the best, and from which to build further $n$-vertices. Unlike other related methods, MDSA is specially designed with pivots to avoid degeneracies which could occur if the simplices were allowed to change shape. For clarity we have included a description of the algorithm as a flow chart Fig. 4.1 together with pictures of the basic simplex operations Fig. 4.2. We have used an implementation of MDSA coded by G. Sandine [12].

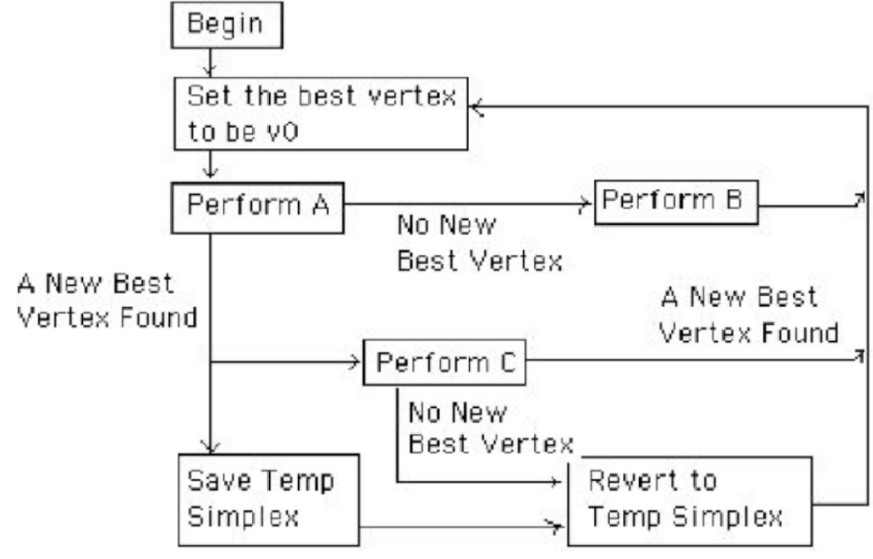

Fig. 4.1. Flow-chart summary of the Torczon - Denis $[10,11]$ MDSA unconstrained optimization algorithm. The simplex operations, A, B, and C, are described pictorially in 2-d in Fig. 4.2. Note that the working "BEST" vertex $v_{0}$ is redefined only at the top of the chart. Notice that the A-B steps of the algorithm are a basic simplex search: reflect about the best point as long as it provides improvement, but otherwise contract towards the best point. C is performed on $v_{0}$ even though there exists by construction at least one better $v^{*}$ to allow for searches beyond $v^{*}$

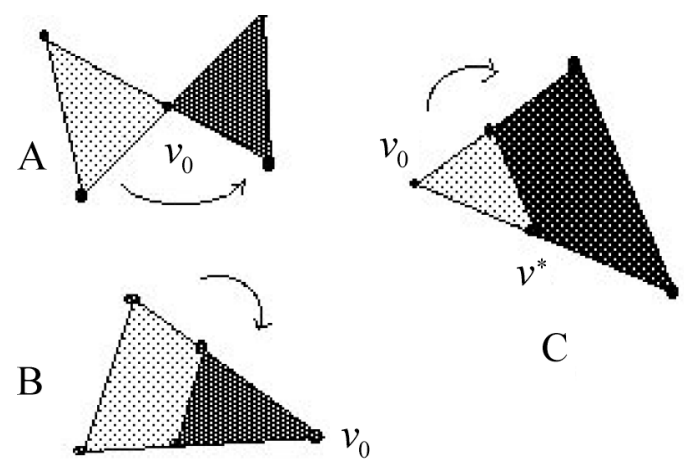

Fig. 4.2. The basic simplex operations performed during iterations of the Torczon Denis $[10,11]$ MDSA unconstrained optimization algorithm, designed to prevent degeneracies. A is reflection through $v_{0}$ by a factor 1. B is contraction towards $v_{0}$ by a factor $\mu<1$. C is expansion away from $v_{0}$ by a factor $\mu>1$. See Fig. 4.1.

For more details on the convergence and specifics of the MDSA method we refer the reader to $[10,11]$ as well as $[12]$. 
4.2. Numerical region evolution and projection. We track the evolution of an initial region, $R(0)$, in terms of evolution of a grid of initial conditions on the boundary of the region, $\partial R(0)$; this is a tracer particle method. There exist more sophisticated methods to track evolution of curves, most notably, the level-set methods [14] which allow for changes of topology of the object in a natural way. The tracer particle method however is extremely easy to implement and sufficient for simple examples in which the topology does not change.

Assuming a fine partition of the arc length of a test object, $\partial R(0)$, we distribute $N$ points $\left\{\left(x_{i}(0), y_{i}(0)\right\}_{i=1}^{N}\right.$ ordered clockwise around the boundary (see Fig. 4.3.). Each initial condition is then integrated forward in time according to the specific model ODE, Eq. (2.1), to create a traced image position, $\left\{\left(x_{i}(t), y_{i}(t)\right\}_{i=1}^{N}\right.$.

Fig. 4.3. Several test objects from which we created simulated data to match. Notice that the grid of initial tracer points are circled around the boundary of the object

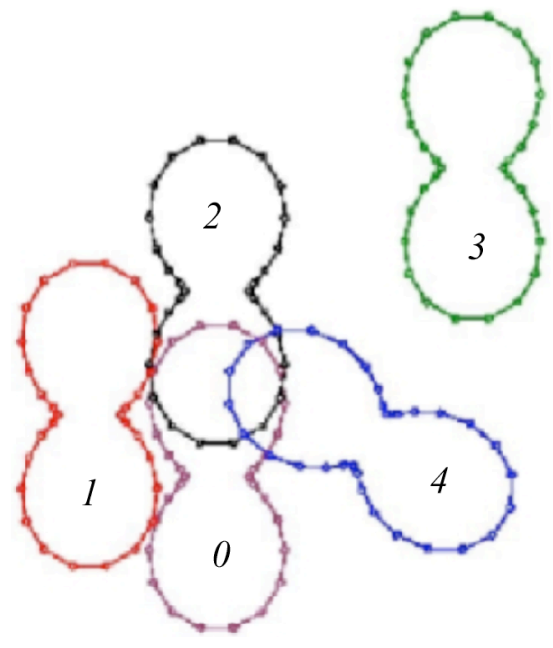

We produce projections of the traced test object, respecting the formal definition, Eq.(2.4), to create discrete data at time- $t, \tilde{D(} t)=\left\{\tilde{d}_{j}(t)\right\}_{j=1}^{p}$, a discrete-space density profile where

$$
\tilde{d}_{j}(t)=\int_{\mathbb{R}^{2} \cap x_{j} \leqslant x \leqslant x_{j+1}} \mathbf{1}_{R(t)}(x, y) d y=\int_{x_{j}}^{x_{j+1}} y_{\text {upper }}(x)-y_{\text {lower }}(x) d x,
$$

Here $\left\{x_{j}\right\}_{j=1}^{p+1}$ refers to the endpoints of the mesh on which the projected data $\tilde{D(t)}$ is sampled by this projection (see Fig. 4.4). Thus, we must create test data sets $\left\{\tilde{D}\left(t_{i}\right)\right\}_{i=1}^{N}$ to

Fig. 4.4. Numerical projection of a region $R(t)$, recorded as $N$-points $\left\{\left(x_{i}(t), y_{i}(t)\right)\right\}_{i=1}^{N}$ around its boundary. Reduction procedure to a density profile $D(t)$ on a grid of $p$-points, by integration is shown. Notice, that we need to take differences of integrations of piecewise linear functions. Maintaining the grid of points in a clockwise order allows us to quickly sort which points are over a given $d_{i}$ grid element of the density projection, and then to carry out the necessary integration differences shown in shading intensities

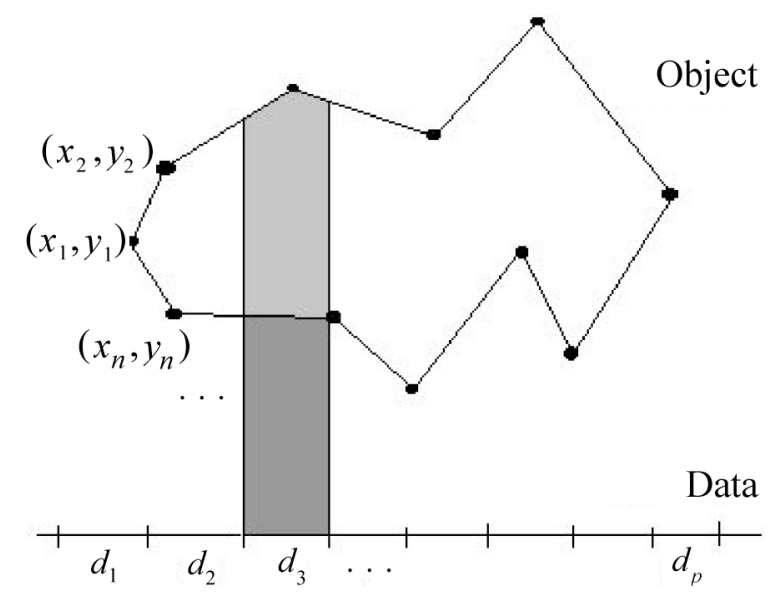

compare to the supplied measured data sets, $\left\{D\left(t_{i}\right)\right\}_{i=1}^{N}$, at times $t_{i}$. Note that $y_{\text {upper }}(x)$ and $y_{\text {lower }}(x)$ are easily calculated since we assume line segments between knots of the object 
data, $\left\{\left(x_{i}(t), y_{i}(t)\right\}_{i=1}^{N}\right.$, where we need to test only that $x_{j}(t) \leqslant x_{i}(t) \leqslant x_{j+1}(t)$, which follows the requirement that we keep the tracers around the boundary in clockwise order.

4.3. Implementation and numerical experiment. Approximating the cost functional (2.8), incorporating evolution of the region $R(0)$ by tracking the tracers on the boundary, and approximating the projection operator as described by Eq. (4.1) we get

$$
J[R(0)] \approx F\left[\left\{x_{i}(0), y_{i}(0)\right\}_{i=1}^{N} ;\left\{D\left(t_{i}\right)\right\}_{i=1}^{N} ; \mathbf{f}_{\mathbf{p}}\right)=\sum_{i=1}^{n}\left(\sum_{j=1}^{p}\left|\tilde{d}_{j}\left(t_{i}\right)-d_{j}\left(t_{i}\right)\right|^{2}\right)^{1 / 2}
$$

We point out that $F$ is a function of the placement of the $N$-points $\left(\left\{\left(x_{i}(0), y_{i}(0)\right\}_{i=1}^{N}\right.\right.$ which it is our job to choose in order to optimize $F$. We consider the projection data $\left\{D\left(t_{i}\right)\right\}_{i=1}^{N}$ and the model vector field, $\mathbf{f}_{\mathbf{p}}$ in $(2.1)$ to be fixed.

\section{Numerical results for radiographic tomography}

With the cost function now well defined in terms of adjustable coefficients, $\left(\left\{x_{i}(0), y_{i}(0)\right\}_{i=1}^{N}\right.$, it is now straightforward to enlist the MDSA to solve our inverse problem. We illustrate the success of this approach with the following concrete example.

Example 5.1. Consider the following shear flow,

$$
\dot{\mathbf{z}}=\mathbf{f}_{\mathbf{p}}(\mathbf{z})=\left(\begin{array}{c}
-\sin (x) \sin (x) \sin (2 y) \\
\sin (2 x) \sin (y) \sin (y)
\end{array}\right)
$$

whose vector field is shown in Fig. 5.1. We have run many experiments involving evolution of test objects under this flow. Several of these test objects are peanut-shaped regions as

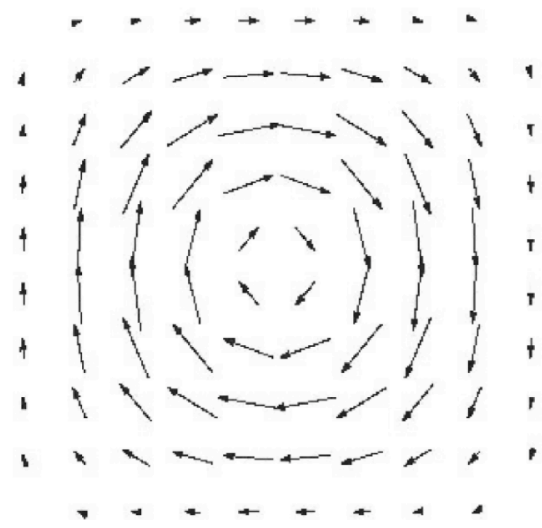

Fig. 5.1. Shear flow Eq. (5.1) used for our presented example numerical inverse problem experiment

depicted in Fig. 4.3. No significant differences in results or problems due to initial placement and orientation were detected. Consider the initial peanut-shaped region shown in the left panel of Fig. 5.2, which distorts under the shear flow as shown in the successive panels as time evolves. A "pseudo" test data set $\left\{D\left(t_{i}\right)\right\}_{i=0}^{p}$ is produced by projecting the distorting region, as defined by Eq. (4.1). This projection data is shown left to right for increasing time in the upper panels of Fig. 5.3. Then we do no know the initial shape, and make initial guesses at the region $R(0)$. After several iterations of the MDSA, we minimize the cost function $F$, Eq. (4.2), with a small computed residual. The fitted region is visually nearly identical to the original peanut, and the projected data is virtually identical. 


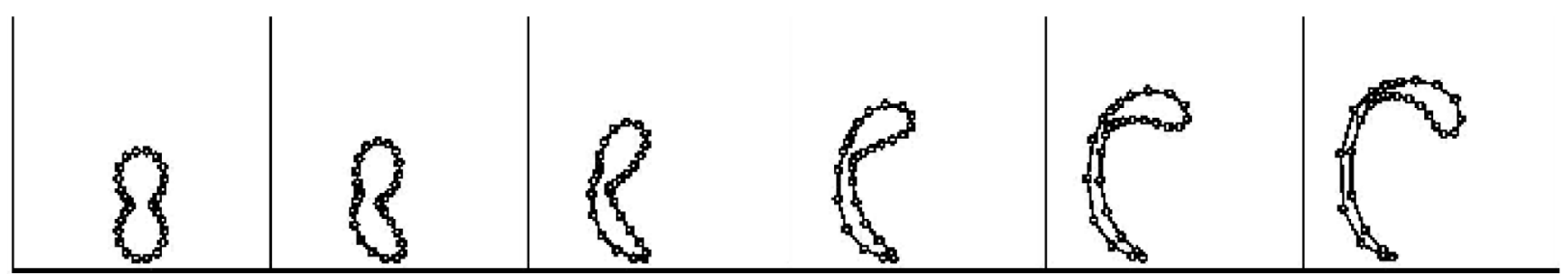

Fig. 5.2. Distortion under a shear flow, Eq. (5.1) of an initial peanut-shaped region, in the left panel, and successive time snapshots $t_{i}$ shown in successive times increasing to the right
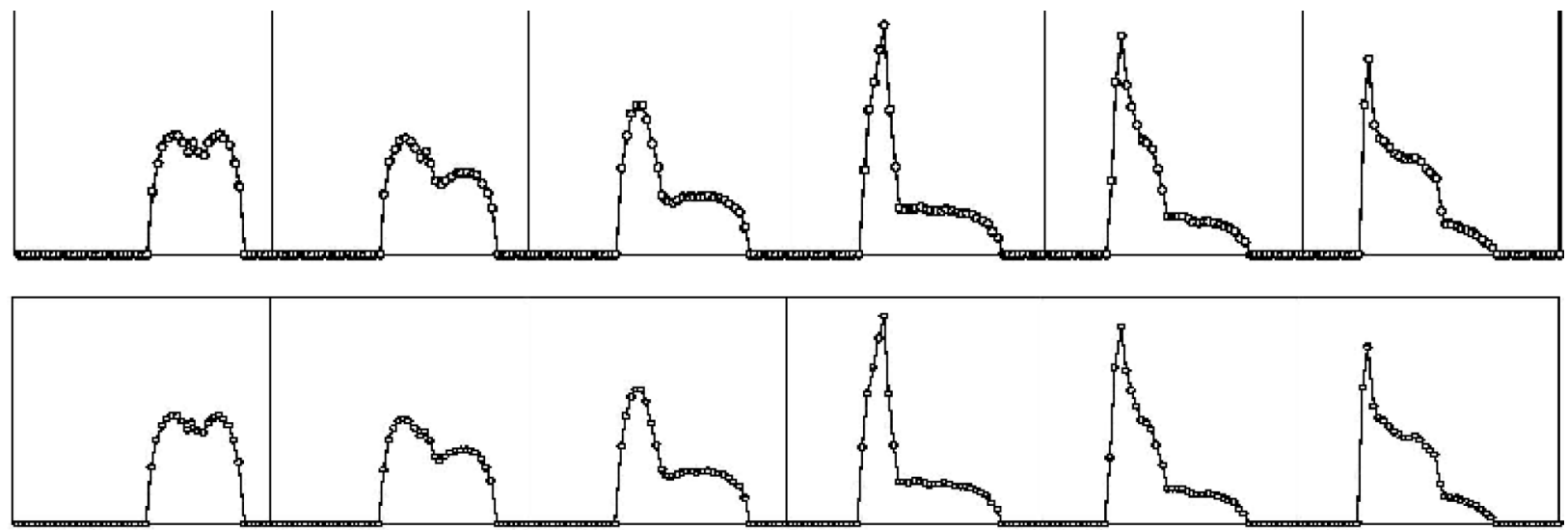

F ig. 5.3. (Top) Left to right, projection data $D\left(t_{i}\right)$ at successive times. (Bottom) Reconstructed data $\tilde{D}\left(t_{i}\right)$ from fitted region which minimizes cost functional, Eq. (4.2). There is no discernible difference, since there is a small measured residual. Likewise, the fitted region $R(0)$ is visually identical (not shown, but appears exactly as in Fig. 5.2)

\section{System identification problem}

We wish to infer parameters (the physics) of a system by observation of data from some unknown simple object. In some cases, one can directly and separately measure parameters, such as how a mass is measured directly on a scale before being added to an experimental dynamical system. However, sometimes parameters must be inferred by observation of the dynamics [6]. A general form of the model is assumed, a parameterized family of vector fields, as stated in Eq. (2.1) where specific parameters such as values of masses, friction, viscosity, etc, are left as unknown parameters, which we must estimate. Thus, we have the radiographic tomography problem together with estimation of parameters.

We may or may not be interested in the form of the evolution in the object. In fact, in another version of the system identification problem, we could assume that we know the initial object is physically reasonable. We assume that we know neither the good $R(0)$ nor the $\mathbf{p}$ in Eq. (2.1). Thus, we write $R_{\mathbf{p}}(t)$ to emphasize a family of evolving objects with identical initial object $R(0)$ governed by parameterized vector fields $\mathbf{f}_{\mathbf{p}}(\mathbf{z})$. Thus we get the test data function,

$$
D_{\mathbf{p}}\left(t_{i}\right) \equiv P\left[R_{\mathbf{p}}\left(t_{i}\right)\right]
$$

which generalizes Eq. (2.9), and from which we generalize Eq. (2.8) to become,

$$
J[R(0) ; \mathbf{p}]=\sum_{i=0}^{n}\left\|\tilde{D_{\mathbf{p}}} \tilde{\left(t_{i}\right)}-D\left(t_{i}\right)\right\|_{L^{1}(\mathbb{R})} .
$$


The numerical techniques for the system identification problem are the straightforward generalization of those for the radiographic tomography problems. For each fixed value of $\mathbf{p}$, projection via Eq. (4.1) is only different implicitly through the specific vector field $\mathbf{f}_{\mathbf{p}}(\mathbf{z})$. Similarly, the cost function Eq. (4.2), $F$ now explicitly depends on the choice of $\mathbf{p}$, and minimization of $F$ with a small residual indicates a good family of models was chosen. The minimization procedure is still through MDSA, but now we allow $\mathbf{p}$ to vary in our code. Since MDSA is gradient free, there are no variations $\partial F \partial \mathbf{p}$ to compute.

Example 6.1. Consider the following shear flow in which we have eight unknown parameters to fit,

$$
\dot{\mathbf{z}}=\mathbf{f}_{\mathbf{p}}(\mathbf{z})=\left(\begin{array}{c}
-p_{0} \sin (x) \sin (x) \sin (2 y)+p_{1}+p_{2} x+p_{3} y \\
p_{4} \sin (2 x) \sin (y) \sin (y)+p_{5}+p_{6} x+p_{7} y
\end{array}\right),
$$

where the domain for the dynamics is $[0, \pi] \times[0, \pi]$. In this flow we introduce a simple closed curve which we study with single view radiography while it undergoes the dynamics described by the above equations. The curve is a polygon defined by the following vertices:

$$
x_{j}=x_{c}+r \cos (2 \pi j / n,), \quad y_{j}=y_{c}+r \sin (2 \pi j / n), \quad r=0.13\left(1+4 \sin ^{2}(2 \pi j / n)\right),
$$

where $x_{c}=\pi / 2, y_{c}=\pi / 4, j=0,1, \ldots, n-1$, and $n=24$. The object is the peanut polygon of $n=24$ sides.

Our goal is to determine the 48 initial coordinates $\left(\left\{x_{i}, y_{i}\right\}, i=1, \ldots, 24\right)$ plus the additional eight parameters $\left(p_{i}, i=1, \ldots, 8\right)$ which determine the dynamics using only the 1-dimensional radiographs (the mass projection of the object onto the $x$-axis apportioned into $q=30$ bins in each time view) and the knowledge that the dynamics are in the 8dimensional family described by (6.3). We do not use the knowledge of the actual values of the parameters $\left(p_{0}=p_{4}=1\right.$ and $p_{i}=0$ otherwise).

Two measures of merit are used in these studies. First, we define the relative datamatching merit function for $v$ time shots given by

$$
f=\left(\sum_{i=1}^{q v}\left|\tilde{d}_{i}-d_{i}\right|\right) /\left(\sum_{i=1}^{q v}\left|\tilde{d}_{i}\right|\right)
$$

where $\tilde{d}_{i}$ is the actual data, and $d_{i}$ is the projection data of the reconstruction. The second is the object-matching merit function which is given by

$$
g=\frac{\operatorname{area}(O \bigcup R-O \bigcap R)}{2 \operatorname{area}(O)}
$$

where $O$ and $R$ are the object and reconstruction polygons respectively.

Eight experiments were completed for varying number of time shots

$$
v \in\{2,3,4,5,6,7,9,11\} .
$$

In each experiment the object is evolved to the same final time and the data are collected at equally spaced times within this interval. In this study the data is noiseless.

The figures 6.1-6.4 on the following pages show many of the details of the final reconstructions for these test cases. One important parameter, however, is missing from 

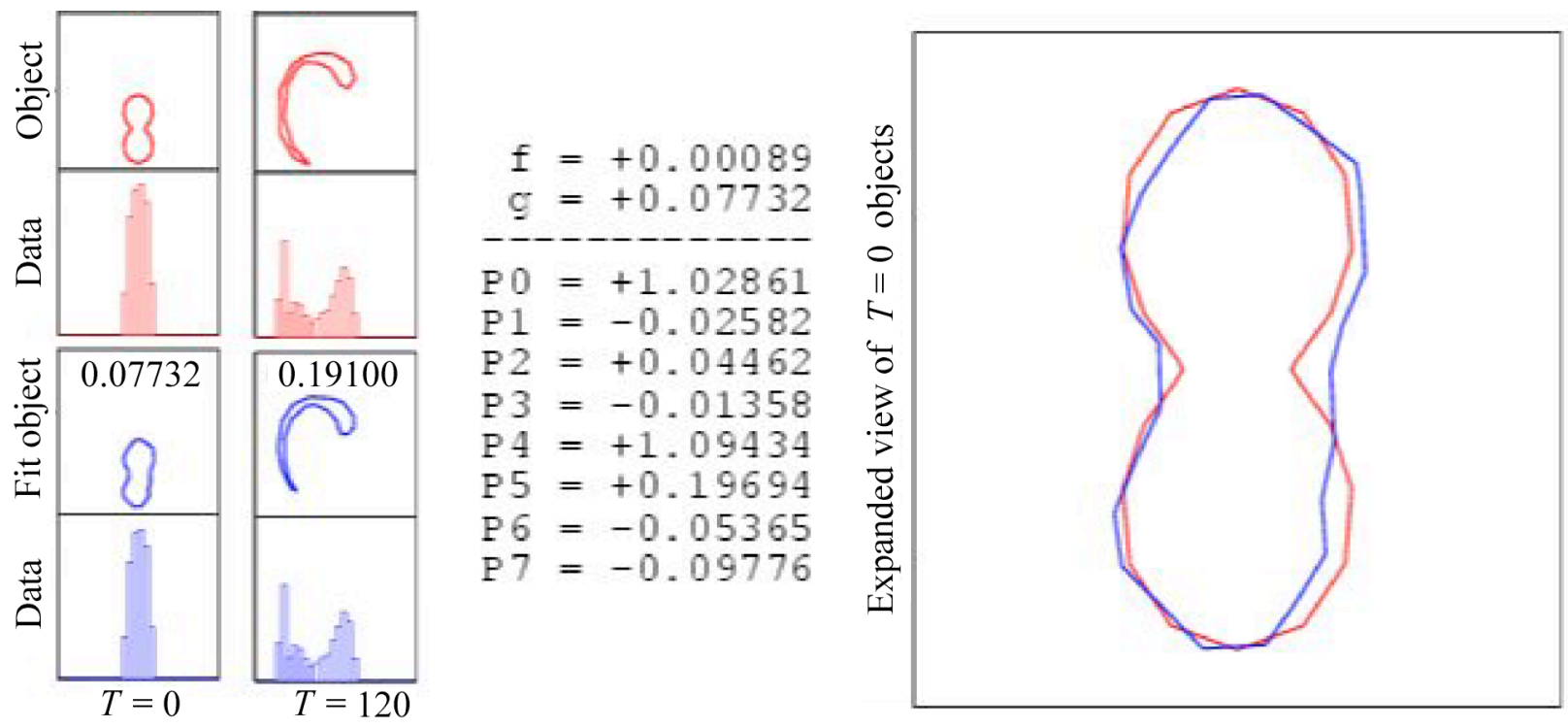

Fig. 6.1. Results for views corresponding to two time shots. (Left-Above) Simulated data evolving in time in the first row. Simulated data density profile available to parameter estimation program. The third row shows the best fitted objects comparable to the first row, with residuals shown. The fourth row shows the best fitted density profiles from row three, and compared to row two. (Right) Zoomed viewed of the initial test object in red and the best fitted object in blue. (Middle) Data sets showing $f$ from (6.4) and $g$ from (6.5), and fitted parameters estimated for (5.1)
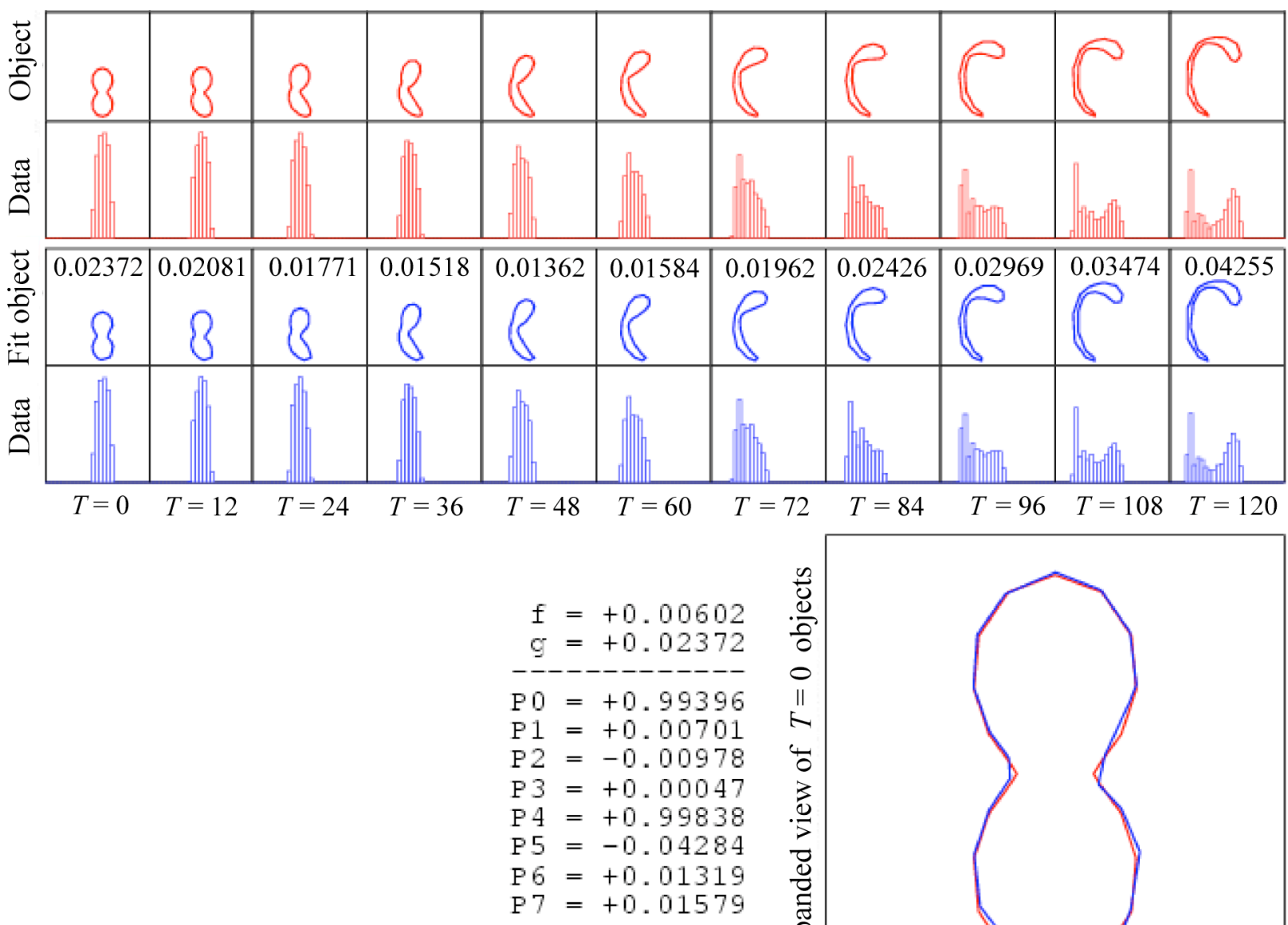

Fig. 6.2. Results for eleven views in time, where results are interpreted as

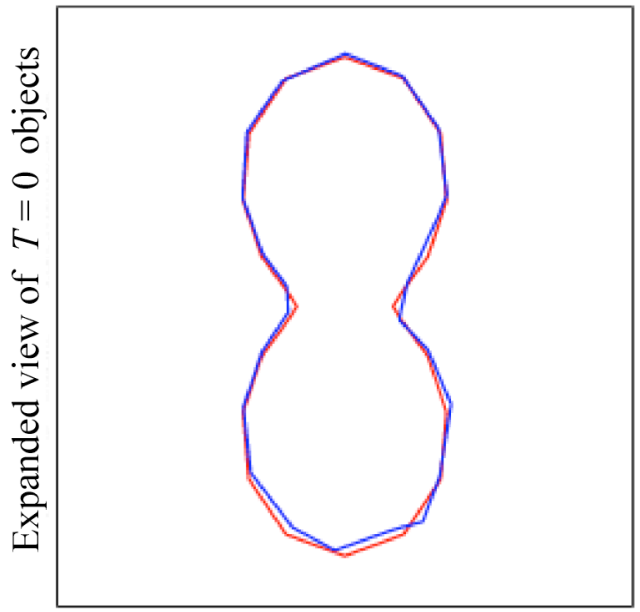

in Fig. 6.1 


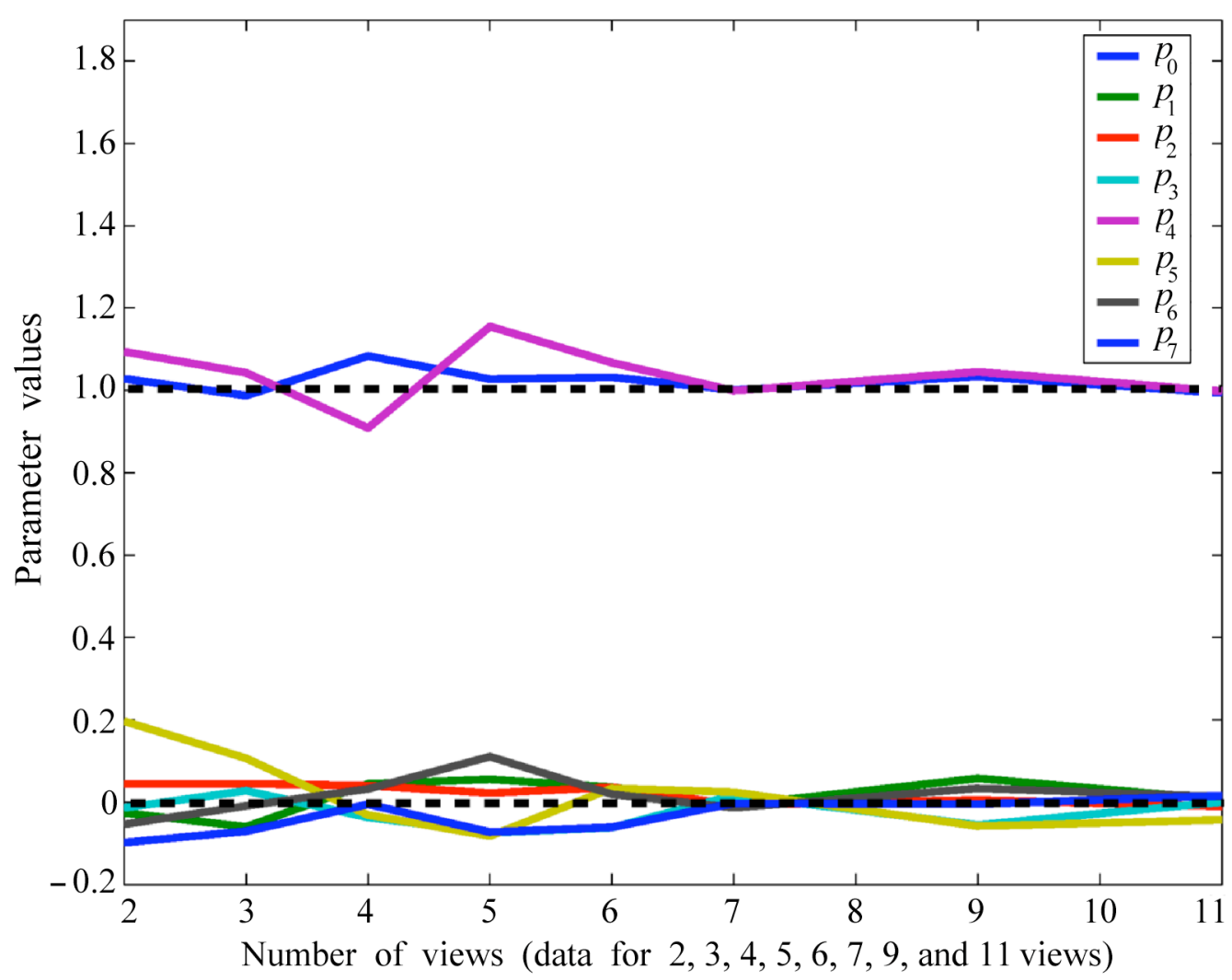

Fig. 6.3. Parameter values in Eq. (5.1) as a function of the number of views

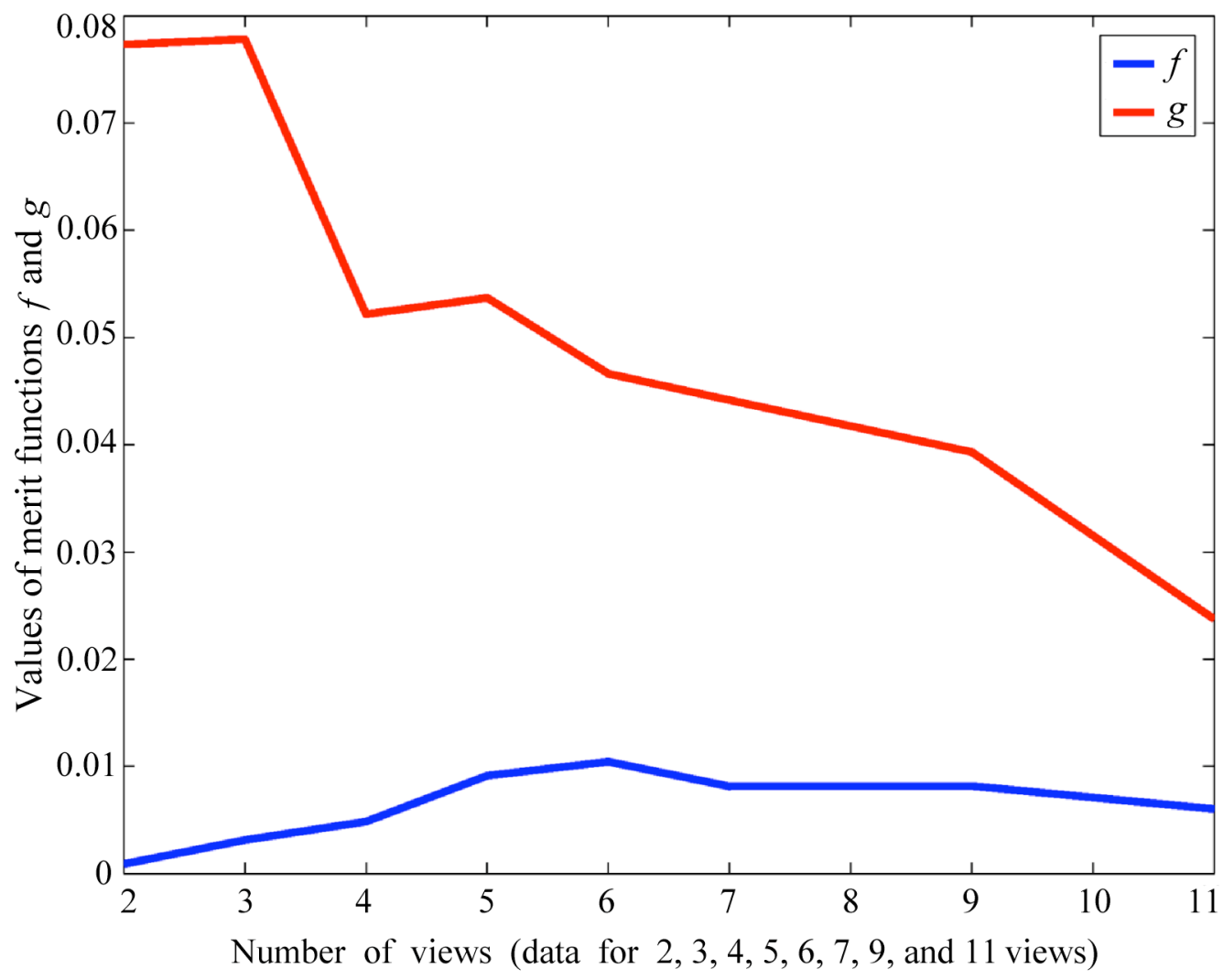

Fig. 6.4. Values of the merit functions, $f$ from Eq. (6.4) and $g$ from Eq. (6.5), as a function of number of views 
the descriptions: the degree of determinacy of the problem. The amount of data useful to the reconstruction in each case is not simply $p v$ because much of the data consist of zero values at the detector. Suppose then that we take the number of relevant data values $N$ to be approximately the number of nonzero data values We have then that $N=\{23,31,41,50,60,70,86,104\}$. Because the number of unknowns for each experiment is 56 (two coordinates for each vertex plus eight parameters in the dynamics), the problem does not become overdetermined until there are at least six time views.

A final figure, Fig. 6.5, displays the object merit $g$ for each computational time step and each experiment set. While this information would not be available in an actual experiment, this plot serves to illustrate how tightly bound our object descriptions are at times intermediate to the sparse data collection.

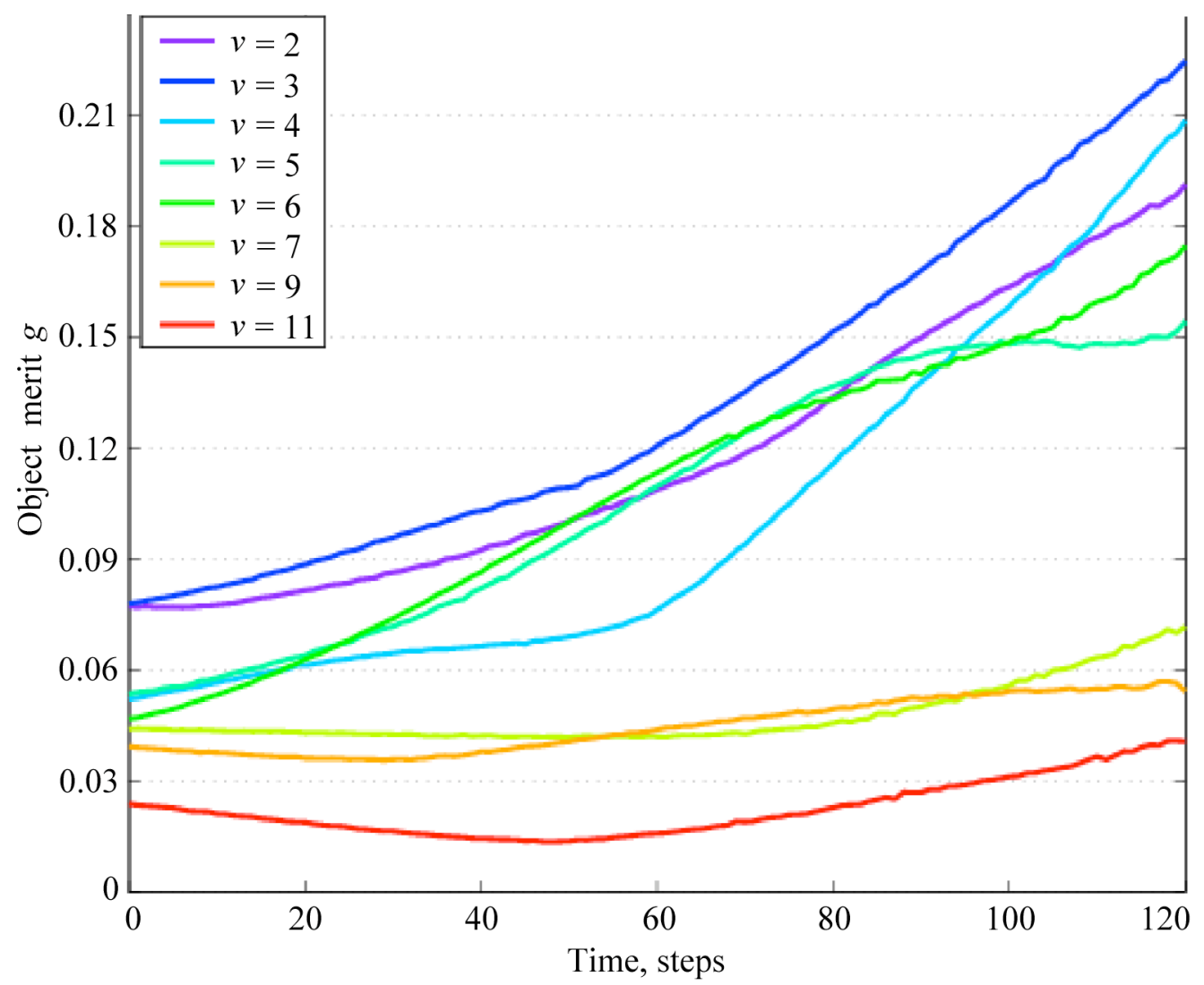

Fig. 6.5. Object merit $g$ in Eq. (6.5) as a function of the number time steps (g is a local in time measure)

We make the following observations:

1. The data can be well matched using any number of time shots. This is no surprise considering the dimensionality, and in the light of Theorem 3.1.

2. The initial and later time object descriptions improve irregularly with increasing number of time shots. Possible exceptions can be due to accidental good guesses.

3. The evolved object descriptions are not always similar in quality throughout a time sequence. $g$ does tend to increase with time, especially for those sequences with the fewest time shots. Often the best object matches occur mid-sequence.

4. With many time shots, when the problem is overdetermined, the object descriptions are excellent. In these simple examples the object descriptions also do not vary significantly from the actual object at intermediate times for which we have no data. 
5. The fit parameters are reasonably well determined for all cases, but do not show a definite convergence trend. The best overall parameter fits do occur for 7 and 11 views. But other cases are similarly good (e.g., $v=2$ ), and some cases fall short of good expectations (e.g., $v=9)$.

\section{References}

1. Y. Shi, W. C. Karl, and D. A. Casstanon, Dynamics tomography using curve evolution with spatiotemporal regularizion, (preprint, 2002).

2. K. R. Vixie and G. L. Sandine, Reconstruction from projections using dynamics: non-stochastic case, (preprint, 2002).

3. K. R. Vixie, Signals and Hidden Information, Ph. D. Dissertation, Portland State University, 2001.

4. N. H. Abel, Magazin for Naturvidenskaberne, Årgang I, Bind2, Christina, (1823), pp. 11-27.

5. E. W. Weisstein, Abel Transform, From MathWorld - A Wolfram Web Resource. http://mathworld. wolfram.com/AbelTransform.html

6. L. Ljung, System Identification: Theory for the User, 2nd Ed., Prentice Hall PTR, 1998.

7. T. Cover and J. A. Thomas, Elements of Information Theory, Wiley-Interscience, New York, 1991.

8. A. Lasota and M. C. Mackey, Chaos, fractals, and noise, 2nd Ed., Springer-Verlag, New York, 1994.

9. E. Bollt, L. Billings, and I. Schwartz, A manifold independent approach to understanding transport in stochastic dynamical systems, Physica D, 173 (2002), no. 3-4, pp. 153-177. Available at http://www.clarkson. edu/ bolltem

10. V. Torczon, Multi-directional search: a direct search algorithm for parallel machines, Ph. D Thesis, Rice University, Houston, TX, 1989.

11. V. Torczon, On the convergence of the multidirectional search algorithm, SIAM J. Optimization, 1 (1991), no. 1.

12. G. L. Sandine, Torczon's and Dennis' multidirectional search algorithm for unconstrained optimization, Informal notes and algorithm, Los Alamos National Laboratory, 2000.

13. R. Mifflin, A superlinearly convergent algorithm for minimization without evaluation of derivatives, Math. Programming, 9 (1975).

14. S. Osher and R.P. Fedkiw, The Level Set Method and Dynamic Implicit Surfaces, Springer-Verlag, New York, 2002.

15. D. Aeyel, textitGeneric observability of differentiable systems, SIAM Journal of Control and Optimization, 19 (1981), no. 5, pp. 595-603.

16. M. Balde and P. Jouan, Genericity and observability of control-affine systems, ESAIM: Control, Optimization, and Calculus of Variations, (1998), no. 3, pp. 345-359.

17. J. W. Polderman and J. C. Willems, Introduction to mathematical systems theory: a behavioral approach, Texts in Applied Mathematics, 26 (1998).

18. T. Sauer, J. A. Yorke, and M. Casdagli, Embedology, Journal of Statistical Physics, 65 (1991), no. 3/4, pp. 579-616.

19. E. D. Sontag, Mathematical control theory: deterministic finite dimensional systems, Texts in Applied Mathematics, 6 (1998).

20. J.Stark, Delay embeddings for forced systems. I: Deterministic forcings, Journal of Nonlinear Science, 9 (1999), pp. 255-332.

21. F. Takens, Detecting strange attractors in turbulence, Dynamic Days at Warwick, number 898 in Lectue Notes in Mathematics. Springer Verlag, 1981.

22. K. R. Vixie and G. L. Sandine, Reconstruction from projections using dynamics: non-stochastic case, LANL e-print archive, 2001. http://arXiv.org

23. N.P. Willis and Y. Bresler, Optimalscan design for time varying tomographic imaging I: Theoretical analysis and fundamental limitations, IEEE Trans. Image Processing, pp. 642-653, May 1995.

24. N.P. Willis and Y. Bresler, Optimal scan design for time varying tomographic imaging II: Efficient design and experimental validation, IEEE Trans. Image Processing, pp. 654-666, May 1995. 